\title{
BEST CONSTANTS FOR CERTAIN MULTILINEAR INTEGRAL OPERATORS
}

\author{
ÁRPÁD BÉNYI AND CHOONGHONG (TADAHIRO) OH
}

Received 7 December 2004; Revised 8 March 2005; Accepted 27 March 2005

We provide explicit formulas in terms of the special function gamma for the best constants in nontensorial multilinear extensions of some classical integral inequalities due to Hilbert, Hardy, and Hardy-Littlewood-Pólya.

Copyright (C 2006 Á. Bényi and C. (Tadahiro) Oh. This is an open access article distributed under the Creative Commons Attribution License, which permits unrestricted use, distribution, and reproduction in any medium, provided the original work is properly cited.

\section{Introduction and statement of results}

In his lectures on integral equations, Hilbert initiated the study of maxima of bilinear and multilinear forms. The case $p=2$ in the following inequality is nowadays known as "Hilbert's double series theorem."

$$
\begin{aligned}
& \text { If } p>1, p^{\prime}=p /(p-1), \sum_{m=1}^{\infty} a_{m}^{p} \leq A \text {, and } \sum_{n=1}^{\infty} b_{n}^{p^{\prime}} \leq B \text {, then } \\
& \qquad \sum_{m=1}^{\infty} \sum_{n=1}^{\infty} \frac{a_{m} b_{n}}{m+n} \leq \pi \csc \left(\frac{\pi}{p}\right) A^{1 / p} B^{1 / p^{\prime}}
\end{aligned}
$$

Hilbert's proof, apart from the determination of the best possible constant $\pi \csc (\pi / p)$, was published by Weyl [7]. The calculation of the constant, and the integral analogue of Hilbert's double series theorem (for $p=2$ ) are due to Schur [6]. The generalizations to other $p^{\prime} s$ of both the discrete and integral versions of this result were discovered later on by Hardy and Riesz and published by Hardy in [4]. The statement of the integral analogue of the theorem above is the following.

If $p>1, p^{\prime}=p /(p-1), \int_{0}^{\infty} f^{p}(x) d x \leq F, \int_{0}^{\infty} g p^{\prime}(y) d y \leq G$, then

$$
\iint_{0}^{\infty} \frac{f(x) g(y)}{x+y} d x d y \leq \pi \csc \left(\frac{\pi}{p}\right) F^{1 / p} G^{1 / p^{\prime}} .
$$

Other proofs and generalizations in different directions were given by several authors; see the book by Hardy et al. [5, page 227]. Applications of Hilbert's theorem range from the 
2 Best constants for certain multilinear integral operators

theory of analytic functions to results about the moments of real variable functions [5, pages 236-247].

Let now $K: \mathbf{R}_{+}^{m} \rightarrow \mathbf{R}$ be a measurable kernel such that

$$
C_{m}=\int_{0}^{\infty} \cdots \int_{0}^{\infty}\left|K\left(y_{1}, \ldots, y_{m}\right)\right| y_{1}^{-1 / p_{1}} \cdots y_{m}^{-1 / p_{m}} d y_{1} \cdots d y_{m}<\infty ;
$$

here, $1<p_{1}, p_{2}, \ldots, p_{m}<\infty$ are some arbitrary (fixed) indices. The $m$-linear operator $T$ is defined via

$$
T\left(f_{1}, \ldots, f_{m}\right)(x)=\int_{0}^{\infty} \cdots \int_{0}^{\infty} K\left(y_{1}, \ldots, y_{m}\right) f_{1}\left(x y_{1}\right) \cdots f_{m}\left(x y_{m}\right) d y_{1} \cdots d y_{m},
$$

where $x>0$ and $f_{1}, \ldots, f_{m}$ are measurable functions on $\mathbf{R}_{+}=[0, \infty)$. Note that $T$ is in fact an integral operator having a homogeneous kernel $\tilde{K}$ of degree $-m$,

$$
T\left(f_{1}, \ldots, f_{m}\right)(x)=\int_{0}^{\infty} \cdots \int_{0}^{\infty} \tilde{K}\left(x, y_{1}, \ldots, y_{m}\right) f_{1}\left(y_{1}\right) \cdots f_{m}\left(y_{m}\right) d y_{1} \cdots d y_{m},
$$

where $\tilde{K}\left(x, y_{1}, \ldots, y_{m}\right)=x^{-m} K\left(x^{-1} y_{1}, \ldots, x^{-1} y_{m}\right)$. Condition (1.3) can be rewritten as

$$
C_{m}=\int_{0}^{\infty} \cdots \int_{0}^{\infty}\left|\tilde{K}\left(1, y_{1}, \ldots, y_{m}\right)\right| y_{1}^{-1 / p_{1}} \cdots y_{m}^{-1 / p_{m}} d y_{1} \cdots d y_{m}<\infty .
$$

We remark also that, if $p_{0}>1$ is such that $1 / p_{0}+1 / p_{1}+\cdots+1 / p_{m}=1$, then

$$
C_{m}=\int_{0}^{\infty} \cdots \int_{0}^{\infty}\left|\tilde{K}\left(x, 1, y_{2}, \ldots, y_{m}\right)\right| x^{-1 / p_{0}} y_{2}^{-1 / p_{2}} \cdots y_{m}^{-1 / p_{m}} d x d y_{2} \cdots d y_{m}
$$

In particular, (1.6) and (1.7) imply that, if $\tilde{K}$ is symmetric with respect to the variables $x, y_{1}, \ldots, y_{m}$, then $C_{m}$ is a symmetric expression of the indices $p_{0}, p_{1}, \ldots, p_{m}$.

The goal of this paper is twofold: to give a proof of the multilinear extension of the integral inequality (1.2) and to provide some interesting applications of this result.

Theorem 1.1. Let $m \geq 1$ and $1<p, p_{1}, \ldots, p_{m}<\infty$ be such that $1 / p_{1}+1 / p_{2}+\cdots+1 / p_{m}=$ $1 / p$. Then

$$
\left\|T\left(f_{1}, \ldots, f_{m}\right)\right\|_{L^{p}\left(\mathbf{R}_{+}\right)} \leq C_{m}\left\|f_{1}\right\|_{L^{p_{1}}\left(\mathbf{R}_{+}\right)} \cdots\left\|f_{m}\right\|_{L^{p^{m}}\left(\mathbf{R}_{+}\right)},
$$

where $C_{m}$ is the constant defined by (1.3) or (1.6). Moreover, if $K\left(y_{1}, \ldots, y_{m}\right) \geq 0$ for all $y_{1}, \ldots, y_{m} \geq 0$, then the constant $C_{m}$ is the best possible in (1.8).

A less general version of Theorem 1.1, which assumes the kernel to be a positive function and which does not determine the best constant, can be found in [5]. There are many proofs of inequality (1.8). For the sake of completeness, we will recall one which to us seems to be the easiest and which is inspired by an idea of Schur [6] in the linear case; see also [5, page 230]. The determination of the best constant, however, is much more interesting. In this work, a strong emphasis is placed on obtaining explicit formulas, in terms of the special gamma function $\Gamma$, of the best constants in multilinear extensions of certain inequalities due to Hilbert, Hardy, and Hardy-Littlewood-Pólya which do not 
seem to be in the literature. These formulas are presented in Section 3. We prove the main result, Theorem 1.1, in the following section. The elementary, yet nontrivial, proof is a nice application of the Dominated Convergence theorem.

Remark 1.2. An alternate way of finding an upper bound for the norm of a positive multilinear operator is via the so called multilinear Schur test in the work of Grafakos and Torres [2, Theorem 1]. In particular, for an $m$-linear operator $T$ with positive and symmetric kernel on $\mathbf{R}_{+}^{m}$, the multilinear Schur test gives the following implication.

If for all $B>A>0$, there exist measurable functions $u_{1}, \ldots, u_{m}, w$ on $\mathbf{R}_{+}$with $0<$ $u_{1}, \ldots, u_{m}, w<\infty$ a.e. such that

$$
T\left(u_{1}^{p_{1}^{\prime}}, \ldots, u_{m}^{p_{m}^{\prime}}\right) \leq B w^{p^{\prime}} \quad \text { a.e., }
$$

then $T$ is a bounded operator from $L^{p_{1}}\left(\mathbf{R}_{+}\right) \times \cdots \times L^{p_{m}}\left(\mathbf{R}_{+}\right)$into $L^{p}\left(\mathbf{R}_{+}\right)$, with norm less than or equal to $A$.

In the case of the multilinear nontensorial extension of the Hilbert operator $P_{\neg \otimes}$ (see Section 3.1 for its definition), it was shown in [2] that an appropriate choice of the functions $u_{1}, \ldots, u_{m}, w$ gives in fact equality in (1.9). Furthermore, it is easy to see that the same choice of weights $u_{j}\left(y_{j}\right)=y_{j}^{-1 / p_{j} p_{j}^{\prime}}, w(x)=x^{-1 / p p^{\prime}}, 1 \leq j \leq m$, gives equality in (1.9) for all $m$-linear operators $T$ of the form (1.4). In this case $B=C_{m}$, where $C_{m}$ is defined by (1.3). Therefore, by the implication above, we see that the operator norm $\|T\| \leq C_{m}$. We would like to point out, however, that in order to show $\|T\|=C_{m}$ one needs to either trace back where equality holds in the inequalities proving the main result of [2] or go through a similar computation to the one presented here in the next section.

\section{Proof of Theorem 1.1}

We let $g \in L^{p^{\prime}}\left(\mathbf{R}_{+}\right), 1 / p+1 / p^{\prime}=1$ and denote by $\langle\cdot, \cdot\rangle$ the dual $\left(L^{p}, L^{p^{\prime}}\right)$ pairing. For simplicity, we will write $L^{p}$ for $L^{p}\left(\mathbf{R}_{+}\right)$. Note that, for $i=1, \ldots, m, \int_{0}^{\infty}\left|f_{i}\left(x y_{i}\right)\right|^{p_{i}} d x=$ $y_{i}^{-1} \int_{0}^{\infty}\left|f_{i}(x)\right|^{p_{i}} d x$. Using this fact and Hölder's inequality, we obtain the following sequence of inequalities:

$$
\begin{aligned}
& \left|\left\langle T\left(f_{1}, \ldots, f_{m}\right), g\right\rangle\right| \\
& \quad \leq \int_{0}^{\infty} \cdots \int_{0}^{\infty}\left|K\left(y_{1}, \ldots, y_{m}\right)\right| \int_{0}^{\infty}|g(x)|\left|f_{1}\left(x y_{1}\right)\right| \cdots\left|f_{m}\left(x y_{m}\right)\right| d x d y_{1} \cdots d y_{m} \\
& \quad \leq \int_{0}^{\infty} \cdots \int_{0}^{\infty}\left|K\left(y_{1}, \ldots, y_{m}\right)\right|\|g\|_{L^{p^{\prime}}} \prod_{i=1}^{m}\left(\int_{0}^{\infty}\left|f_{i}\left(x y_{i}\right)\right|^{p_{i}} d x\right)^{1 / p_{i}} d y_{1} \cdots d y_{m} \\
& \quad=C_{m}\|g\|_{L^{p^{\prime}}}|| f_{1}\left\|_{L^{p_{1}}} \cdots\right\| f_{m} \|_{L^{p_{m}}} .
\end{aligned}
$$

This proves the first part of our theorem.

For the second part, we will show that if the kernel $K$ is nonnegative, then the operator norm $\|T\|$ of $T$ is exactly $C_{m}$. For $n$ a positive integer and $i=1, \ldots, m$, we define the 
4 Best constants for certain multilinear integral operators

sequences of functions $g_{n}, f_{i, n}$ by

$$
g_{n}(x)=x^{-1 / p^{\prime}+1 / p^{\prime} n} \chi_{[0,1]}(x), \quad f_{i, n}(x)=x^{-1 / p_{i}+1 / p_{i} n} \chi_{[0,1]}(x)
$$

Clearly, $\left\|g_{n}\right\|_{L^{p^{\prime}}}^{p^{\prime}}=\left\|f_{i, n}\right\|_{L^{p_{i}}}^{p_{i}}=\left\|g_{n}\right\|_{L^{p^{\prime}}}\left\|f_{1, n}\right\|_{L^{p_{1}}} \cdots\left\|f_{m, n}\right\|_{L^{p m}}=n$. We have

$$
\begin{aligned}
& \left\langle T\left(f_{1, n}, \ldots, f_{m, n}\right), g_{n}\right\rangle \\
& \quad=\int_{0}^{1} x^{-1 / p^{\prime}+1 / p^{\prime} n} \int_{0}^{\infty} \cdots \int_{0}^{\infty} K\left(y_{1}, \ldots, y_{m}\right) f_{1, n}\left(x y_{1}\right) \cdots f_{m, n}\left(x y_{m}\right) d y_{1} \cdots d y_{m} d x \\
& \quad=\int_{0}^{1} x^{-1 / p^{\prime}+1 / p^{\prime} n} \int_{0}^{1 / x} \cdots \int_{0}^{1 / x} K\left(y_{1}, \ldots, y_{m}\right) \prod_{i=1}^{m}\left(x y_{i}\right)^{-1 / p_{i}+1 / p_{i} n} d y_{1} \cdots d y_{m} d x \\
& \quad=\int_{0}^{1} x^{-1+1 / n} \int_{0}^{1 / x} \cdots \int_{0}^{1 / x} K\left(y_{1}, \ldots, y_{m}\right) \prod_{i=1}^{m} y_{i}^{-1 / p_{i}+1 / p_{i} n} d y_{1} \cdots d y_{m} d x \\
& \quad=-n \int_{1}^{\infty}\left(x^{-1 / n}\right)^{\prime}\left(\int_{0}^{x} \cdots \int_{0}^{x} K\left(y_{1}, \ldots, y_{m}\right) \prod_{i=1}^{m} y_{i}^{-1 / p_{i}+1 / p_{i} n} d y_{1} \cdots d y_{m}\right) d x \\
& \quad=n \int_{0}^{1} \cdots \int_{0}^{1} K\left(y_{1}, \ldots, y_{m}\right) \prod_{i=1}^{m} y_{i}^{-1 / p_{i}+1 / p_{i} n} d y_{1} \cdots d y_{m}+\sum_{i=1}^{m} I_{i},
\end{aligned}
$$

or

$$
\begin{aligned}
& \frac{\left\langle T\left(f_{1, n}, \ldots, f_{m, n}\right), g_{n}\right\rangle}{\left\|g_{n}\right\|_{L^{p^{\prime}}}\left\|f_{1, n}\right\|_{L^{p_{1}}} \cdots\left\|f_{m, n}\right\|_{L^{p m}}} \\
& \quad=\int_{0}^{1} \cdots \int_{0}^{1} K\left(y_{1}, \ldots, y_{m}\right) \prod_{i=1}^{m} y_{i}^{-1 / p_{i}+1 / p_{i} n} d y_{1} \cdots d y_{m}+\sum_{i=1}^{m} I_{i} / n .
\end{aligned}
$$

For $i=1, \ldots, m$, we have denoted

$$
I_{i}=n \int_{1}^{\infty} y_{i}^{-1 / n} \int_{0}^{y_{i}} \cdots \int_{0}^{y_{i}} K\left(y_{1}, \ldots, y_{m}\right) \prod_{j=1}^{m} y_{j}^{-1 / p_{j}+1 / p_{j} n} d y_{1} \cdots \widehat{d y_{i}} \cdots d y_{m} d y_{i}
$$

here, $\widehat{d y_{i}}$ means that we do not integrate with respect to the variable $y_{i}$. In the transition from the fourth to the fifth line in the sequence of equalities above we made the change of variables $x \mapsto 1 / x$. The last equality follows from integration by parts and the observation that, if we let

$$
S\left(z_{1}, \ldots, z_{m}\right)=\int_{0}^{z_{1}} \cdots \int_{0}^{z_{m}} K\left(y_{1}, \ldots, y_{m}\right) \prod_{i=1}^{m} y_{i}^{-1 / p_{i}+1 / p_{i} n} d y_{1} \cdots d y_{m}
$$


then

$$
\begin{aligned}
\frac{d}{d x} S(x, \ldots, x)=\sum_{i=1}^{m} \frac{\partial S}{\partial z_{i}}(x, \ldots, x)=\sum_{i=1}^{m} \int_{0}^{x} \cdots & \int_{0}^{x} K\left(y_{1}, \ldots, \stackrel{(i)}{x}, \ldots, y_{m}\right) x^{-1 / p_{i}+1 / p_{i} n} \\
& \times \prod_{j \neq i} y_{j}^{-1 / p_{j}+1 / p_{j} n} d y_{1} \cdots \widehat{d y_{i}} \cdots d y_{m}
\end{aligned}
$$

where the upper index $(i)$ means that $x$ replaces the variable $y_{i}$ in the $i$ th position. Let now $D_{i}$ denote the domain of integration in the integral $I_{i}$ above, that is,

$$
D_{i}=\left\{\left(y_{1}, \ldots, y_{m}\right) \in \mathbf{R}_{+}^{m}: 1 \leq y_{i}<\infty, 0 \leq y_{j} \leq y_{i}, j \neq i\right\} .
$$

Taking into account that $1 / p_{1}+\cdots+1 / p_{m}=1 / p$, we can bound the integrand of $I_{i} / n$ on $D_{i}$ as follows:

$$
\begin{array}{rl}
y_{i}^{-1 / n} & K\left(y_{1}, \ldots, y_{m}\right) \prod_{j=1}^{m} y_{j}^{-1 / p_{j}+1 / p_{j} n} \leq y_{i}^{-1 / n+1 / p_{1} n+\cdots+1 / p_{m} n} K\left(y_{1}, \ldots, y_{m}\right) \prod_{j=1}^{m} y_{j}^{-1 / p_{j}} \\
= & y_{i}^{-1 / p^{\prime} n} K\left(y_{1}, \ldots, y_{m}\right) \prod_{j=1}^{m} y_{j}^{-1 / p_{j}} \leq K\left(y_{1}, \ldots, y_{m}\right) \prod_{j=1}^{m} y_{j}^{-1 / p_{j}} .
\end{array}
$$

Also, on $[0,1]^{m}$ we obviously have

$$
K\left(y_{1}, \ldots, y_{m}\right) \prod_{j=1}^{m} y_{j}^{-1 / p_{j}+1 / p_{j} n} \leq K\left(y_{1}, \ldots, y_{m}\right) \prod_{j=1}^{m} y_{j}^{-1 / p_{j}} .
$$

Now, assumption (1.3) on the kernel $K$ allows us to use the Dominated Convergence theorem to infer that

$$
\begin{gathered}
\lim _{n \rightarrow \infty} I_{i} / n=\int_{1}^{\infty} \int_{0}^{y_{i}} \cdots \int_{0}^{y_{i}} K\left(y_{1}, \ldots, y_{m}\right) \prod_{j=1}^{m} y_{j}^{-1 / p_{j}} d y_{1} \cdots d y_{m}, \\
\lim _{n \rightarrow \infty} \int_{0}^{1} \cdots \int_{0}^{1} K\left(y_{1}, \ldots, y_{m}\right) \prod_{j=1}^{m} y_{j}^{-1 / p_{j}+1 / p_{j} n} d y_{1} \cdots d y_{m} \\
=\int_{0}^{1} \cdots \int_{0}^{1} K\left(y_{1}, \ldots, y_{m}\right) \prod_{j=1}^{m} y_{j}^{-1 / p_{j}} d y_{1} \cdots d y_{m} .
\end{gathered}
$$

Furthermore, we have

$$
[0,1]^{m} \bigcup\left(\bigcup_{i=1}^{m} D_{i}\right)=\mathbf{R}_{+}^{m}
$$

and for $i, j=1, \ldots, m$, any of the intersection sets $[0,1]^{m} \cap D_{i}, D_{i} \cap D_{j}, i \neq j$, has Lebesgue measure 0 in $\mathbf{R}^{m}$. Consequently, (2.4), (2.11) imply that

$$
\|T\|=\lim _{n \rightarrow \infty} \frac{\left\langle T\left(f_{1, n}, \ldots, f_{m, n}\right), g_{n}\right\rangle}{\left\|g_{n}\right\|_{L^{p^{\prime}}}|| f_{1, n}\left\|_{L^{p}} \cdots\right\| f_{m, n} \|_{L^{p m}}}=C_{m} .
$$

The proof is now complete. 
6 Best constants for certain multilinear integral operators

\section{Applications}

In this section we wish to revisit some important inequalities due to Hilbert, HardyLittlewood-Pólya, and Hardy. We will discuss the possible multilinear extensions of these inequalities and provide formulas for the best constants in a closed form via the gamma function. The formulas we present here do not seem to be in the literature. Let us first recall a few basic facts about the gamma and beta functions. For more details and further references on special functions, see the book by Andrews et al. [1]. For a complex number $z$ with $\operatorname{Re} z>0$ define

$$
\Gamma(z)=\int_{0}^{\infty} t^{z-1} e^{-t} d t
$$

It follows from the definition that the gamma function $\Gamma(z)$ is analytic in the right halfplane $\operatorname{Re} z>0$. Two fundamental properties of the gamma function are that

$$
\Gamma(z+1)=z \Gamma(z), \quad \Gamma(z) \Gamma(1-z)=\frac{\pi}{\sin (\pi z)} .
$$

In particular, we also have $\Gamma(n)=(n-1)$ ! for all positive integers $n$. The second formula above is known under the name of Euler's reflection formula. Next we define the beta function. For $z$ and $w$ complex numbers with positive real parts,

$$
B(z, w)=\int_{0}^{1} t^{z-1}(1-t)^{w-1} d t
$$

We have the following relationship between the gamma and beta functions:

$$
B(z, w)=\frac{\Gamma(z) \Gamma(w)}{\Gamma(z+w)} .
$$

3.1. Hilbert's operator. Consider the linear operator

$$
P f(x)=\int_{0}^{\infty} \frac{f(y)}{x+y} d y
$$

with the kernel $\tilde{K}^{(1)}(x, y)=1 /(x+y)$ which is symmetric in the variables $x, y$ and homogeneous of degree -1 . By duality, we see that the integral analogue of Hilbert's theorem (1.2) is equivalent to the best constant inequality

$$
\|P f\|_{L^{p}\left(\mathbf{R}_{+}\right)} \leq \pi \csc \left(\frac{\pi}{p}\right)\|f\|_{L^{p}\left(\mathbf{R}_{+}\right)} .
$$

The operator $P$ is usually referred to as Hilbert's operator and inequality (3.6) as Hilbert's inequality. On our quest for multilinear extensions of (3.6) we would like to preserve the features of the operator $P$. In particular, we wish to extend its kernel $\widetilde{K}^{(1)}$ to kernels $\tilde{K}^{(m)}$ that fit the framework of our main result, Theorem 1.1. One such extension is provided 
by the $m$-linear operator

$$
P_{\otimes}\left(f_{1}, \ldots, f_{m}\right)(x)=\int_{0}^{\infty} \cdots \int_{0}^{\infty} \frac{f_{1}\left(y_{1}\right) \cdots f_{m}\left(y_{m}\right)}{\left(x+y_{1}\right) \cdots\left(x+y_{m}\right)} d y_{1} \cdots d y_{m}
$$

having the kernel

$$
\widetilde{K}_{\otimes}^{(m)}=\frac{1}{\left(x+y_{1}\right) \cdots\left(x+y_{m}\right)}=\prod_{i=1}^{m} \tilde{K}^{(1)}\left(x, y_{i}\right)
$$

Although $\widetilde{K}_{\otimes}^{(m)}$ is symmetric $x, y_{1}, \ldots, y_{m}$ and homogeneous of degree $-m$, and thus it preserves the properties satisfied by its one dimensional counterpart $\widetilde{K}^{(1)}$, the multilinear extension $P_{\otimes}$ is not very interesting due to its tensorial character. Throughout the remaining of this paper, we let $1<p, p_{1}, \ldots, p_{m}<\infty$ be such that $1 / p=1 / p_{1}+\cdots 1 / p_{m}$, and denote by $p_{0}=p^{\prime}$ the dual exponent of $p$. We will also write $L^{p}$ for $L^{p}\left(\mathbf{R}_{+}\right)$. Since

$$
P_{\otimes}\left(f_{1}, \ldots, f_{m}\right)=(P \otimes \cdots \otimes P)\left(f_{1}, \ldots, f_{m}\right)=P\left(f_{1}\right) \cdots P\left(f_{m}\right),
$$

using Hölder's inequality and (3.6), we obviously have

$$
\left\|P_{\otimes}\left(f_{1}, \ldots, f_{m}\right)\right\|_{L^{p}} \leq \pi^{m} \prod_{i=1}^{m} \csc \left(\frac{\pi}{p_{i}}\right)\left\|f_{1}\right\|_{L^{p_{1}}} \cdots\left\|f_{m}\right\|_{L^{p_{m}}} .
$$

The constant on the right (the operator norm $\left\|P_{\otimes}\right\|$ of $P_{\otimes}$ ) is the best possible.

A much more interesting situation arises when we consider the nontensorial extension

$$
P_{\neg \otimes}\left(f_{1}, \ldots, f_{m}\right)(x)=\int_{0}^{\infty} \cdots \int_{0}^{\infty} \frac{f_{1}\left(y_{1}\right) \cdots f_{m}\left(y_{m}\right)}{\left(x+y_{1}+\cdots+y_{m}\right)^{m}} d y_{1} \cdots d y_{m}
$$

having the (symmetric in variables $x, y_{1}, \ldots, y_{m}$ and homogeneous of degree $-m$ ) kernel

$$
\tilde{K}_{\neg \otimes}^{(m)}=\frac{1}{\left(x+y_{1}+\cdots+y_{m}\right)^{m}} .
$$

Noting that $\tilde{K}_{\neg \otimes}^{(m)} \leq \tilde{K}_{\otimes}^{(m)}$, we know that the operator $P_{\neg \otimes}$ is bounded from $L^{p_{1}} \times \cdots \times L^{p_{m}}$ into $L^{p}$. Nevertheless, the a priori constant $\left\|P_{\otimes}\right\|$ is not the best possible in this case.

Claim 3.1. The following best constant inequality holds:

$$
\left\|P_{\neg \otimes}\left(f_{1}, \ldots, f_{m}\right)\right\|_{L^{p}} \leq \frac{1}{(m-1) !} \prod_{i=0}^{m} \Gamma\left(\frac{1}{p_{i}^{\prime}}\right)\left\|f_{1}\right\|_{L^{p_{1}}} \cdots\left\|f_{m}\right\|_{L^{p^{m}}} .
$$

Recall that, due to the homogeneity and symmetry in the variables $x, y_{1}, \ldots, y_{m}$ of $\widetilde{K}_{\neg \otimes}^{(m)}$, the formula that computes the norm of the corresponding operator defined by (3.11) must be a symmetric expression in $p_{0}, p_{1}, \ldots, p_{m}$; see the comments following (1.6). This is indeed the case in Claim 3.1. Observe that the best "tensorial" constant $\left\|P_{\otimes}\right\|$ is symmetric only in $p_{1}, p_{2}, \ldots, p_{m}$, in general. 
8 Best constants for certain multilinear integral operators

Proof. By Theorem 1.1, the best constant is given by

$$
C_{m}=\int_{0}^{\infty} \cdots \int_{0}^{\infty}\left(1+y_{1}+\cdots+y_{m}\right)^{-m} y_{1}^{-1 / p_{1}} \cdots y_{m}^{-1 / p_{m}} d y_{1} \cdots d y_{m} .
$$

Let us denote the integral on the right by $I_{m}\left(m, 1 / p_{1}, \ldots, 1 / p_{m}\right)$. By making the change of variables $y_{m}=\left(1+y_{1}+\cdots+y_{m-1}\right) t$ and integrating first with respect to $d t$, we get

$$
I_{m}\left(m, 1 / p_{1}, \ldots, 1 / p_{m}\right)=\int_{0}^{\infty}(1+t)^{-m} t^{-1 / p_{m}} d t I_{m-1}\left(m-1 / p_{m}^{\prime}, 1 / p_{1}, \ldots, 1 / p_{m-1}\right) .
$$

Observe that, if we make the change of variables $t+1=1 / s$,

$$
\int_{0}^{\infty}(1+t)^{\alpha} t^{\beta} d t=\int_{0}^{1} s^{-\alpha-\beta-2}(1-s)^{\beta} d s=B(-\alpha-\beta-1, \beta+1) .
$$

Therefore, if we recall the relationship between the beta and gamma functions, we obtain

$$
\begin{gathered}
\int_{0}^{\infty}(1+t)^{-m} t^{-1 / p_{m}} d t=\frac{\Gamma\left(m-1 / p_{m}^{\prime}\right) \Gamma\left(1 / p_{m}^{\prime}\right)}{\Gamma(m)}, \\
I_{m}\left(m, 1 / p_{1}, \ldots, 1 / p_{m}\right)=\frac{\Gamma\left(m-1 / p_{m}^{\prime}\right) \Gamma\left(1 / p_{m}^{\prime}\right)}{\Gamma(m)} I_{m-1}\left(m-1 / p_{m}^{\prime}, 1 / p_{1}, \ldots, 1 / p_{m-1}\right) .
\end{gathered}
$$

By a simple induction argument, we obtain from this recurrence that

$$
I_{m}\left(m, 1 / p_{1}, \ldots, 1 / p_{m}\right)=\frac{\Gamma\left(m-1 / p_{m}^{\prime}-\cdots-1 / p_{1}^{\prime}\right) \Gamma\left(1 / p_{m}^{\prime}\right) \cdots \Gamma\left(1 / p_{1}^{\prime}\right)}{\Gamma(m)} .
$$

Due to the relation on the exponents, the latter equality simplifies exactly to

$$
C_{m}=\frac{1}{(m-1) !} \prod_{i=0}^{m} \Gamma\left(\frac{1}{p_{i}^{\prime}}\right)
$$

Remark 3.2. One can construct other multilinear extensions of $P$. For example, consider the $m$-linear operator

$$
P_{m}\left(f_{1}, \ldots, f_{m}\right)(x)=\int_{0}^{\infty} \cdots \int_{0}^{\infty} \frac{f_{1}\left(y_{1}\right) \cdots f_{m}\left(y_{m}\right)}{\left(x+y_{1} \cdots y_{m}\right)^{m}} d y_{1} \cdots d y_{m} .
$$

For $m=1$ we clearly recover $P$ from $P_{m}$. Note, however, that the kernel of $P_{m}$ is not symmetric with respect to $x, y_{1}, \ldots, y_{m}$, and we do not have anymore the a priori boundedness of the operator on products of Lebesgue spaces, since $P_{m}$ and $P_{\otimes}$ are not comparable. As such, the operator $P_{m}$ does not fall under the scope of our main result, Theorem 1.1. For the remainder of the paper, we will avoid any further discussion about multilinear operators that are arbitrary extensions (i.e., which do not preserve the features) of the classical linear operators considered. 
3.2. Hardy-Littlewood-Pólya's operator. We let $Q$ denote Hardy-Littlewood-Pólya’s linear operator defined by

$$
Q f(x)=\int_{0}^{\infty} \frac{f(y)}{\max (x, y)} d y .
$$

Its $m$-linear nontensorial extension is

$$
Q_{\neg \otimes}\left(f_{1}, \ldots, f_{m}\right)(x)=\int_{0}^{\infty} \cdots \int_{0}^{\infty} \frac{f_{1}\left(y_{1}\right) \cdots f_{m}\left(y_{m}\right)}{\left[\max \left(x, y_{1}, \ldots, y_{m}\right)\right]^{m}} d y_{1} \cdots d y_{m} .
$$

Claim 3.3. The following best constant inequality holds:

$$
\left\|Q_{\neg \otimes}\left(f_{1}, \ldots, f_{m}\right)\right\|_{L^{p}} \leq\left(\sum_{i=0}^{m} \prod_{j=0, j \neq i}^{m} p_{j}^{\prime}\right)\left\|f_{1}\right\|_{L^{p_{1}}} \cdots\left\|f_{m}\right\|_{L^{p m}} .
$$

Proof. With the notation in the proof of Theorem 1.1, we can decompose $\mathbf{R}_{+}^{m}$ into the union $[0,1]^{m} \cup\left(\bigcup_{i=1}^{m} D_{i}\right)$ so that the interiors of the sets in this union are pariwise disjoint. Furthermore, the best constant in the inequality we want to prove is

$$
\begin{aligned}
C_{m} & =\int_{0}^{\infty} \cdots \int_{0}^{\infty}\left[\max \left(1, y_{1}, \ldots, y_{m}\right)\right]^{-m} y_{1}^{-1 / p_{1}} \cdots y_{m}^{-1 / p_{m}} d y_{1} \cdots d y_{m} \\
& =\int_{[0,1]^{m}} y_{1}^{-1 / p_{1}} \cdots y_{m}^{-1 / p_{m}} d y_{1} \cdots d y_{m}+\sum_{i=1}^{m} J_{i}
\end{aligned}
$$

where

$$
J_{i}=\int_{1}^{\infty} \int_{0}^{y_{i}} \cdots \int_{0}^{y_{i}} y_{1}^{-1 / p_{1}} \cdots y_{i}^{-m-1 / p_{i}} \cdots y_{m}^{-1 / p_{m}} d y_{1} \cdots \widehat{d y_{i}} \cdots d y_{m} d y_{i} .
$$

Now,

$$
\int_{[0,1]^{m}} y_{1}^{-1 / p_{1}} \cdots y_{m}^{-1 / p_{m}} d y_{1} \cdots d y_{m}=\left.\prod_{j=1}^{m} \frac{y_{j}^{1-1 / p_{j}}}{1-1 / p_{j}}\right|_{0} ^{1}=\prod_{j=1}^{m} p_{j}^{\prime}
$$

and (recall that $\left.p_{0}^{\prime}=p\right)$

$$
\begin{aligned}
J_{i} & =\left.\int_{1}^{\infty} y_{i}^{-m-1 / p_{i}} \prod_{j=1, j \neq i}^{m} \frac{y_{j}^{1-1 / p_{j}}}{1-1 / p_{j}}\right|_{0} ^{y_{i}} d y_{i}=\prod_{j=1, j \neq i}^{m} p_{j}^{\prime} \int_{1}^{\infty} y_{i}^{-1-1 / p_{1}-\cdots-1 / p_{m}} d y_{i} \\
& =\left.\prod_{j=1, j \neq i}^{m} p_{j}^{\prime} \frac{y_{i}^{-1 / p_{1}-\cdots-1 / p_{m}}}{-1 / p_{1}-\cdots-1 / p_{m}}\right|_{1} ^{\infty}=\prod_{j=0, j \neq i}^{m} p_{j}^{\prime} .
\end{aligned}
$$

By summing up, we obtain the desired result. 
In particular, for $m=1$, we recover the best constant in the $\left(L^{p}, L^{p}\right)$ inequality satisfied by the operator $Q, C_{1}=p+p^{\prime}=p^{2} /(p-1)$; see [5, page 254]. Furthermore, for the $m$ linear tensorial extension of $Q$,

$$
Q_{\otimes}\left(f_{1}, \ldots, f_{m}\right)(x)=\int_{0}^{\infty} \cdots \int_{0}^{\infty} \frac{f_{1}\left(y_{1}\right) \cdots f_{m}\left(y_{m}\right)}{\max \left(x, y_{1}\right) \cdots \max \left(x, y_{m}\right)} d y_{1} \cdots d y_{m}
$$

the best constant inequality is

$$
\left\|Q_{\otimes}\left(f_{1}, \ldots, f_{m}\right)\right\|_{L^{p}} \leq \prod_{i=1}^{m} \frac{p_{i}^{2}}{p_{i}-1}\left\|f_{1}\right\|_{L^{p_{1}}} \cdots\left\|f_{m}\right\|_{L^{p m}} .
$$

3.3. Hardy's operator. In his attempts to simplify the proofs known at the time of Hilbert's double series theorem, Hardy introduced in [3] the operator

$$
R f(x)=\frac{1}{x} \int_{0}^{x} f(y) d y
$$

and proved that it is bounded from $L^{p}$ into $L^{p}$ with best constant $p /(p-1)$. Unlike the kernels of the operators $P, Q$ considered before, the kernel $\tilde{k}^{(1)}(x, y)=(1 / x) \chi_{[0, x]}(y)$ of $R$ is not symmetric with respect to the variables $x, y$ (to emphasize this difference, we use now the lower case letter $k$ ). Here, $\chi_{I}$ denotes the characteristic function of the set $I$. The $m$-linear tensorial extension

$$
R_{\otimes}\left(f_{1}, \ldots, f_{m}\right)(x)=\frac{1}{x^{m}} \int_{0}^{x} \cdots \int_{0}^{x} f_{1}\left(y_{1}\right) \cdots f_{m}\left(y_{m}\right) d y_{1} \cdots d y_{m}
$$

is then bounded from $L^{p_{1}} \times \cdots \times L^{p_{m}}$ into $L^{p}$, and the best constant is $\prod_{i=1}^{m} p_{i} /\left(p_{i}-\right.$ 1). Note that the kernel $\tilde{k}_{\otimes}^{(m)}$ of $R_{\otimes}$ is still positive and homogeneous of degree $-m$, as required in the hypothesis of Theorem 1.1. In our search for an appropriate nontensorial multilinear extension of $R$, we observed that we could also write $\tilde{k}^{(1)}(x, y)=$ $(1 / x) \chi_{[0,1]}(y / x)=(1 / x) \chi_{[1+y / x, \infty)}(2)$. This simple observation has suggested that we define the nontensorial extension via

$$
R_{\neg \otimes}\left(f_{1}, \ldots, f_{m}\right)(x)=\frac{1}{x^{m}} \int_{0}^{\infty} \cdots \int_{0}^{\infty} \chi_{[0, x]}\left(y_{1}+\cdots+y_{m}\right) f_{1}\left(y_{1}\right) \cdots f_{m}\left(y_{m}\right) d y_{1} \cdots d y_{m} .
$$

It is worth noting that, as in the previous examples, the kernel of the nontensorial extension satisfies $\widetilde{k}_{\neg \otimes}^{(m)} \leq \widetilde{k}_{\otimes}^{(m)}$. We therefore have the a priori boundedness of $R_{\neg \otimes}$ from $L^{p_{1}} \times \cdots \times L^{p_{m}}$ into $L^{p}$. However, since $\tilde{k}_{\neg \otimes}^{(m)}$ is symmetric in the variables $y_{1}, \ldots, y_{m}$ but not in $x$, we expect the operator norm of the nontensorial extension to be symmetric in $p_{1}, \ldots p_{m}$ but not in $p_{0}=p^{\prime}$ (the index that "corresponds" to $x$ ).

Claim 3.4. The following best constant inequality holds:

$$
\left\|R_{\neg \otimes}\left(f_{1}, \ldots, f_{m}\right)\right\|_{L^{p}} \leq \frac{\prod_{i=1}^{m} \Gamma\left(1 / p_{i}^{\prime}\right)}{\Gamma\left(m+1 / p_{0}\right)}\left\|f_{1}\right\|_{L^{p_{1}}} \cdots\left\|f_{m}\right\|_{L^{p_{m}}} .
$$


Proof. By Theorem 1.1, the best constant is given by

$$
\begin{aligned}
C_{m} & =\int_{0}^{\infty} \cdots \int_{0}^{\infty} \chi_{[0,1]}\left(y_{1}+\cdots+y_{m}\right) y_{1}^{-1 / p_{1}} \cdots y_{m}^{-1 / p_{m}} d y_{1} \cdots d y_{m} \\
& =\int_{0}^{1} \int_{0}^{1-y_{1}} \cdots \int_{0}^{1-y_{1}-\cdots-y_{m-1}} y_{1}^{-1 / p_{1}} \cdots y_{m}^{-1 / p_{m}} d y_{m} \cdots d y_{1} \\
& =p_{m}^{\prime} \int_{0}^{1} \int_{0}^{1-y_{1}} \cdots \int_{0}^{1-\sum_{k=1}^{m-2} y_{k}} y_{1}^{-1 / p_{1}} \cdots y_{m-1}^{-1 / p_{m-1}}\left(1-\sum_{k=1}^{m-1} y_{k}\right)^{1 / p_{m}^{\prime}} d y_{m-1} \cdots d y_{1} .
\end{aligned}
$$

In the last integral, we make the substitution $y_{m-1}=t_{m-1}\left(1-\sum_{k=1}^{m-2} y_{k}\right)$ and integrate first with respect to $d t_{m-1}$. This allows us to simplify to an integral over only $m-2$ variables multiplied by the integral in $t_{m-1}, \int_{0}^{1} t_{m-1}^{-1 / p_{m-1}}\left(1-t_{m-1}\right)^{1 / p_{m}^{\prime}} d t_{m-1}=B\left(1 / p_{m}^{\prime}, 1+1 / p_{m}^{\prime}\right)$. More precisely, we have

$$
\begin{aligned}
C_{m}= & p_{m}^{\prime} \frac{\Gamma\left(1 / p_{m-1}^{\prime}\right) \Gamma\left(1+1 / p_{m}^{\prime}\right)}{\Gamma\left(1+1 / p_{m-1}^{\prime}+1 / p_{m}^{\prime}\right)} \\
& \times \int_{0}^{1} \int_{0}^{1-y_{1}} \cdots \int_{0}^{1-\sum_{k=1}^{m-3} y_{k}} y_{1}^{-1 / p_{1}} \cdots y_{m-2}^{-1 / p_{m-2}}\left(1-\sum_{k=1}^{m-2} y_{k}\right)^{1 / p_{m-1}^{\prime}+1 / p_{m}^{\prime}} d y_{m-2} \cdots d y_{1} .
\end{aligned}
$$

Since, $p_{m}^{\prime} \Gamma\left(1+1 / p_{m}^{\prime}\right)=\Gamma\left(1 / p_{m}^{\prime}\right)$, by letting successively $y_{m-2}=t_{m-2}\left(1-\sum_{k=1}^{m-3} y_{k}\right), \ldots$, $y_{2}=t_{1}\left(1-y_{1}\right)$ and integrating with respect to $d t_{m-2}, \ldots, d t_{1}$ respectively, we obtain

$$
C_{m}=\frac{\prod_{i=1}^{m} \Gamma\left(1 / p_{i}^{\prime}\right)}{\Gamma\left(1+\sum_{i=1}^{m}\left(1 / p_{i}^{\prime}\right)\right)} .
$$

Since, $\sum_{i=1}^{m} 1 / p_{i}^{\prime}=m-1 / p$, the desired result follows.

Remark 3.5. The integral inequalities we considered have corresponding discrete versions. For example, the $m$-linear discrete versions of Hilbert's and Hardy-LittlewoodPólya's inequalities are, respectively,

$$
\begin{gathered}
\sum_{k=1}^{\infty} \sum_{k_{1}=1}^{\infty} \cdots \sum_{k_{m}=1}^{\infty} \frac{a_{k} a_{k_{1}} \cdots a_{k_{m}}}{\left(k+k_{1}+\cdots+k_{m}\right)^{m}} \leq C_{m}\left(\sum_{k=1}^{\infty} a_{k}^{p^{\prime}}\right)^{1 / p^{\prime}} \prod_{j=1}^{m}\left(\sum_{k_{j}=1}^{\infty} a_{k_{j}}^{p_{j}}\right)^{1 / p_{j}}, \\
\sum_{k=1}^{\infty} \sum_{k_{1}=1}^{\infty} \cdots \sum_{k_{m}=1}^{\infty} \frac{a_{k} a_{k_{1}} \cdots a_{k_{j}}}{\left(\max \left(k, k_{1}, \cdots, k_{m}\right)\right)^{m}} \leq C_{m}^{\prime}\left(\sum_{k=1}^{\infty} a_{k}^{p^{\prime}}\right)^{1 / p^{\prime}} \prod_{j=1}^{m}\left(\sum_{k_{j}=1}^{\infty} a_{k_{j}}^{p_{j}}\right)^{1 / p_{j}} .
\end{gathered}
$$

The constants $C_{m}$ and $C_{m}^{\prime}$ are the ones obtained in Claims 3.1 and 3.3, respectively. The proofs of the discrete versions follow from an appropriate discrete analogue of Theorem 1.1 , but we will not pursue such a result here; the interested reader is referred to [5, page 232] for further details. 


\section{Best constants for certain multilinear integral operators}

\section{Acknowledgment}

We are indebted to the anonymous referees for the many valuable suggestions that improved the quality of our presentation.

\section{References}

[1] G. E. Andrews, R. Askey, and R. Roy, Special Functions, Encyclopedia of Mathematics and Its Applications, vol. 71, Cambridge University Press, Cambridge, 1999.

[2] L. Grafakos and R. H. Torres, A multilinear Schur test and multiplier operators, Journal of Functional Analysis 187 (2001), no. 1, 1-24.

[3] G. H. Hardy, Note on a theorem of Hilbert, Mathematische Zeitschrift 6 (1920), no. 3-4, 314-317.

[4] __ Note on a theorem of Hilbert concerning series of positive terms, Proceedings of the London Mathematical Society 23 (1925), 45-46.

[5] G. H. Hardy, J. E. Littlewood, and G. Pólya, Inequalities, 2nd ed., Cambridge University Press, Cambridge, 1952.

[6] I. Schur, Bemerkungen zur Theorie der beschränkten Bilinearformen mit unendlich vielen veränderlichen, Journal für die Reine und Angewandte Mathematik 140 (1911), 1-28 (German).

[7] H. Weyl, Singülare Integralgleichungen mit besonderer Berücksichtigung des Fourierschen Integraltheorems, Inaugural-Dissertation, Gottingen, 1908.

Árpád Bényi: Department of Mathematics, 516 High Street, Western Washington University, Bellingham, WA 98225-9063, USA

E-mail address: arpad.benyi@wwu.edu

Choonghong (Tadahiro) Oh: Department of Mathematics and Statistics, Lederle GRT,

University of Massachusetts, Amherst, MA 01003-9305, USA

E-mail address: oh@math.umass.edu 